\title{
In Situ
}

Revue des patrimoines

$28 \mid 2016$

Le moulage. Pratiques historiques et regards contemporains

Marmoris Memoriae. Photographies réalisées à l'atelier des moulages des Musées royaux d'art et d'histoire de Bruxelles

Marmoris Memoriae. Photographs taken in the workshop of the royal museums of art and history, Brussels

\section{Martin Declève}

\section{(2)enEdition}

\section{Journals}

Édition électronique

URL : http://journals.openedition.org/insitu/12764

DOI : $10.4000 /$ insitu. 12764

ISSN : 1630-7305

\section{Éditeur}

Ministère de la culture

\section{Référence électronique}

Martin Declève, «Marmoris Memoriae. Photographies réalisées à l'atelier des moulages des Musées royaux d'art et d'histoire de Bruxelles », In Situ [En ligne], 28 | 2016, mis en ligne le 15 mars 2016, consulté le 01 mai 2019. URL : http://journals.openedition.org/insitu/12764 ; DOI : 10.4000/ insitu. 12764

Ce document a été généré automatiquement le 1 mai 2019.

\section{c) (†)}

In Situ Revues des patrimoines est mis à disposition selon les termes de la licence Creative Commons Attribution - Pas d'Utilisation Commerciale - Pas de Modification 4.0 International. 


\title{
Marmoris Memoriae. Photographies réalisées à l'atelier des moulages des Musées royaux d'art et d'histoire de Bruxelles
}

\author{
Marmoris Memoriae. Photographs taken in the workshop of the royal \\ museums of art and history, Brussels
}

Martin Declève

Communication présentée lors des journées d'étude « Le moulage. Pratiques historiques et regards contemporains » organisées par la Cité de l'architecture et du patrimoine et le musée du quai Branly, les 14 et 15 novembre 2012.

1 J'étais occupé au montage d'une exposition présentant une partie des photographies réalisées à l'atelier des moulages des Musées royaux d'art et d'histoire (MRAH), dans le cadre de la manifestation "Parcours d'artistes ", à Bruxelles, lorsque j'appris, grâce à Bernard Van den Driessche, qu'allaient se tenir à Paris deux journées d'étude sur le moulage. Mon travail répondant à l'intitulé de ces journées - en tout cas à sa deuxième proposition, j'entends les « regards contemporains » - je décidai d'y assister. C'est encore à Bernard Van den Driessche que je dois, après qu'il eut fait mention de mon travail au cours de la discussion qui venait clore la première matinée, l'invitation qui me fut faite par les organisateurs de présenter mon travail.

2 Le texte qui suit a été rédigé à l'occasion de cette exposition, les illustrations sont celles des photographies que l'on pouvait y voir. Il n'est pas aisé d'écrire soi-même sur son propre travail ; pas aisé, parfois même peut-être pas souhaitable. Que l'on prenne donc ces quelques lignes non pour des commentaires, encore moins pour des explications, mais pour une tentative d'éclaircissement des hypothèses de travail, des rêveries qui m'animent. Ce projet n'en est, j'espère, qu'à son commencement. Bien qu'il n'ait été 
montré jusqu'ici que très partiellement lors d'une exposition à caractère intime, les réactions des spectateurs, parfois inattendues, et les échanges avec eux, ont été pour moi très instructifs.

Une exposition, intitulée « Dans le creux du plâtre, l'exil des Illustres ", a depuis vu le jour en novembre 2014, au sein de la nouvelle gypsothèque de l'université de Genève, en collaboration avec Lorenz Baumer, professeur d'archéologie classique. Outre le fait d'exposer, c'est également la production d'images que je souhaiterais continuer afin de compléter ainsi les séries entamées - portraits de philosophes, d'empereurs, etc. - à l'occasion de séances de prises de vues dans d'autres ateliers de moulages, au premier rang desquels figure l'atelier de moulages de la Réunion des musées nationaux, à SaintDenis où je ne désespère pas d'aller travailler un jour. Mais photographier des moules masses encombrantes, lourdes et pourtant si fragiles, délicates à manipuler - n'est pas facile et nécessite l'aide de professionnels, membres de l'atelier souvent déjà débordés de travail par ailleurs. J'en remercie d'autant plus vivement Mme Nele Strobbe, directrice de l'atelier des moulages des Musées royaux d'art et d'histoire (MRAH), ainsi que tout le personnel de l'atelier sans qui ce travail n'eut pas été possible. Toute ma reconnaissance va également à M. Michel Draguet, alors directeur par intérim des MRAH, pour l'enthousiasme et le soutien qu'il ne cessa de manifester envers mon travail.

4 Je ne sais pas avec précision ni quand ni comment m'est venue l'idée de photographier des moules à bon creux de l'atelier des moulages des Musées royaux d'art et d'histoire de Bruxelles. Je me souviens que l'endroit, l'atelier, a retenu mon attention dès que j'ai appris son existence, et cela n'était sans doute pas désintéressé par rapport à ma pratique de la photographie. Il est donc resté ainsi - comme « un lieu où il faudrait que j'aille voir un jour » - dans un coin de mon esprit, jusqu'à ce que je me décide à en pousser la porte. J'avais apporté mon équipement et je pus réaliser un essai dès cette première visite; il s'agissait d'un moule en silicone représentant une jeune femme, peut-être Juliette Récamier. Il était déposé sur une table entre les rayons de la réserve près de deux autres pièces, dans le but didactique de montrer aux visiteurs la différence entre un moule à bon creux, un moule en gélatine et un moule en silicone, les trois techniques principales toujours en usage à l'atelier et ailleurs. Ce premier essai, bien que très éloigné du résultat auquel j'arriverais après plusieurs mois de recherche sur le plan de l'éclairage et de mises au point techniques, s'avéra toutefois concluant quant à l'intuition de départ: contrairement à l'œil, l'objectif de l'appareil photographique restituait une image qui paraissait positive, non pas négative - j'emploie cette comparaison, qui a une longue histoire, par commodité -, les creux devenaient saillies, la lumière coulée dans le moule recréait tout un jeu d'ombres et d'éclats qui, bien qu'inversé, aboutissait à un positif qui, d'une tout autre facture que la photographie d'un moulage, n'avait d'autre matérialité que celle de l'image. Ce que les Anciens, sans doute, auraient appelé un " simulacre ».

Pour des raisons que l'on comprendra par la suite, mon choix s'est arrêté, parmi l'immense collection de l'atelier, sur les moules à bon creux de bustes antiques. On nomme aussi ces derniers " moules à pièces ", appellation plus moderne qui nous prive de l'association poétique avec les mânes, nom par lequel les Romains invoquaient l'âme d'un défunt, peut-être par euphémisme, car il signifie littéralement les "Bons». Que les Romains aient utilisé une expression au pluriel pour désigner ce qui, même après la mort, paraît relever de l'individualité, intrigue encore. On explique souvent cette curiosité - qui n'est sans doute pas que linguistique - par le recours à la catégorie du collectif : les âmes, dépouillées de leur personnalité, ne seraient conçues qu'en masse. Les inscriptions 
funéraires antiques s'adressent pourtant aux mânes « d'un tel ou d'un tel » et il semble plus probable que le pluriel n'est employé ici qu'en conformité avec la multiplicité des restes, particulièrement les os. Qui a déjà démonté un moule à bon creux n'a pu qu'être frappé par la ressemblance entre les pièces qui le constituent et des os : des osselets pour certaines, des os aux formes étranges pour d'autres, des os souvent blanchis et lisses pareils à ceux que rejettent les flots.

\section{Rudis et sine imagine tellus (Appia restituta)}

6 L'idée de commencer cette exposition par un sujet qui pourrait paraitre étranger à l'ensemble - ce qu'il reste des tombeaux de la via Appia - s'est imposée lorsque j'ai trouvé comment la réaliser matériellement. Qu'il y ait, pour les Anciens, et plus particulièrement pour les Romains, un lien entre les premières représentations figurées et la mort - le portrait comme substitut magique à la disparition d'un être vivant - toutes leurs légendes relatives à l'origine de la représentation figurée - peinture et sculpture - l'attestent, tout comme leur environnement quotidien, depuis les tombeaux qui bordaient les routes jusqu'aux portraits des ancêtres qui décoraient la première pièce de leur demeure. Le poète latin Ovide, dans ses Métamorphoses, clôt d'ailleurs le récit de l'apparition de l'humanité par ces deux vers splendides:

Sic modo quae fuerat rudis et sine imagine tellus

Induit ignotas hominum conversa figuras.

" Ainsi la terre qui jusque-là était restée informe et dépourvue d'images

se revêtit, par ce changement, des figures des hommes auparavant inconnues. "

Pour un Romain, qui ignorait Lascaux et Chauvet, la première image était celle d'un visage. De visage, sur les tombeaux de la via Appia, il n'y en a plus - si ce n'est quelques mauvaises copies - et leurs niches sont vides. Les lourdes dalles de basalte ont mieux résisté au temps que le marbre et le visiteur qui s'y rend pour la première fois, enchanté par le cadre bucolique, s'étonne toutefois, face à ces monticules de briques, de l'état de délabrement de ce que son guide lui décrit comme étant des tombeaux romains. C'est cette analogie de forme, ou plutôt d'informe, avec les moules fermés que vous verrez dans la première pièce qui m'a d'abord décidé à photographier ces tombeaux. Cependant, la première façon adoptée pour les montrer en relation avec les images de moules imprimés qu'ils étaient sur un papier classique - me laissait insatisfait et j'avais fini par abandonner l'idée. Entretemps, à Carrare, j'avais fait découper deux plaques de marbre de grand format sur lesquelles j'entendais imprimer certaines des images que vous verrez imprimées sur verre. Ce projet passa également à la trappe lorsque j'eus réalisé que ce n'était pas des portraits mais bien les tombeaux qui devaient être imprimés sur ce support, matériau dont ils avaient été dépouillés au cours des siècles. Je fis donc à nouveau découper les deux plaques, cette fois en douze carrés de petit format afin que, présentés très proches les uns des autres, ils créent un concentré qui, à mes yeux, intensifie la perception et donne au regard qui s'y pose une densité étrangère au lieu luimême ou au souvenir que l'on peut en avoir. Ainsi cette série, au sous-titre quelque peu ironique, nous place face à l'absence de l'image tout en initiant une remontée vers elle. 


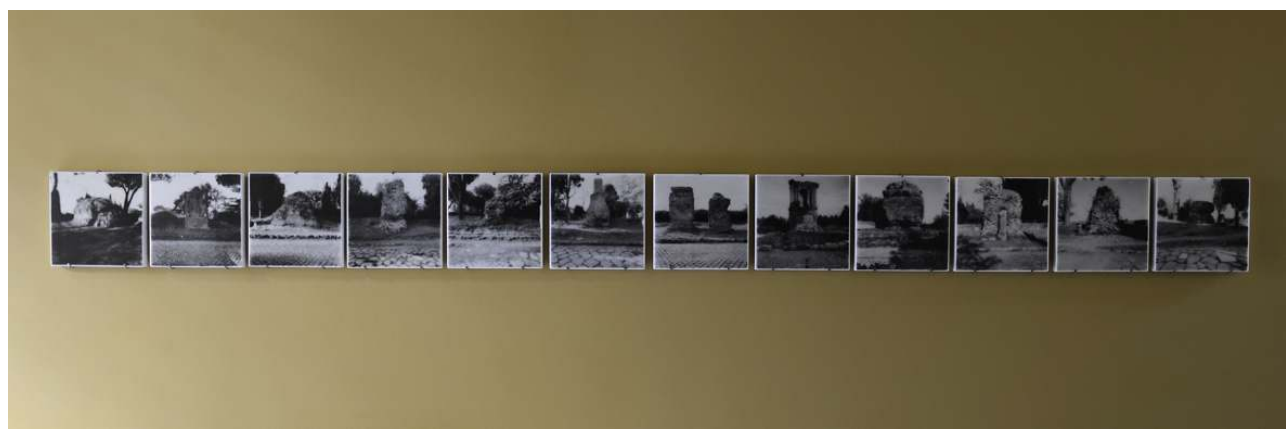

Rudis et sine imagine tellus (Appia restituta), « une terre informe et dépourvue d'images (la via Appia restaurée) ». Photographie, 12 x émulsion sur marbre de Carrare, 20 × $20 \mathrm{~cm}$.

(c) Martin Declève.

\section{Albae umbrae}

Les moules à bon creux sont constitués d'une multitude de pièces en plâtre coulées sur l'original ou, le plus souvent, sur une première copie ou "modèle " (on parle alors de "surmoule »). Si cela n'était pas le cas, l’opération du démoulage serait bien évidemment impossible. Il est nécessaire, afin de maintenir ces pièces - parfois fort nombreuses ensemble, de les enchâsser dans une chape, elle aussi en plâtre, elle aussi démontable, mais constituée de quelques parties seulement, entre six et dix généralement pour les bustes. Si vous vous baladez dans les allées de la réserve de l'atelier des moulages, ce sont ces chapes que vous verrez, par dizaines, allongées sur d'énormes étagères en bois. Allongées car il est toujours risqué de les redresser, des pièces à l'intérieur pouvant se désolidariser de l'ensemble et tomber en dépit du fait que la chape soit solidement ficelée ou, suivant une manière de faire, abandonnée aujourd'hui, maintenue à l'aide de bandes de toile de jute enduites de plâtre presque pareilles à celles dont on bande un membre fracturé. Souvent centenaires, ces moules, en comparaison de ceux réalisés avec des matières synthétiques modernes, comme le silicone, qui a tendance à rétrécir après quelques années, restent les meilleurs garants d'une empreinte exacte, tout en étant fragiles. Les moules les plus abîmés, les plus restaurés, sont ceux dont a été tiré un grand nombre d'épreuves. Au commencement de mon travail, je ne pensais pas photographier les moules ainsi, fermés. Ce n'est qu'après quelques séances que leur beauté et leur mystère me sont apparus. Leur surface mate, rugueuse, parsemée de coulées de plâtre, présente aux jointures des failles au tracé surprenant qui laisse parfois deviner le profil du visage qu'ils renferment. Leur allure mal dégrossie, pareille à celle d'une ombre projetée, a donné son titre à la série. La curiosité de qui les voit est attisée par le numéro qu'ils portent, incisé ou peint, par un nom aussi qui se laisse parfois entrapercevoir sur un morceau de papier kraft ou sur une étiquette, et qui ne manquera pas de faire venir à l'esprit une image, un visage. 
Figure 2

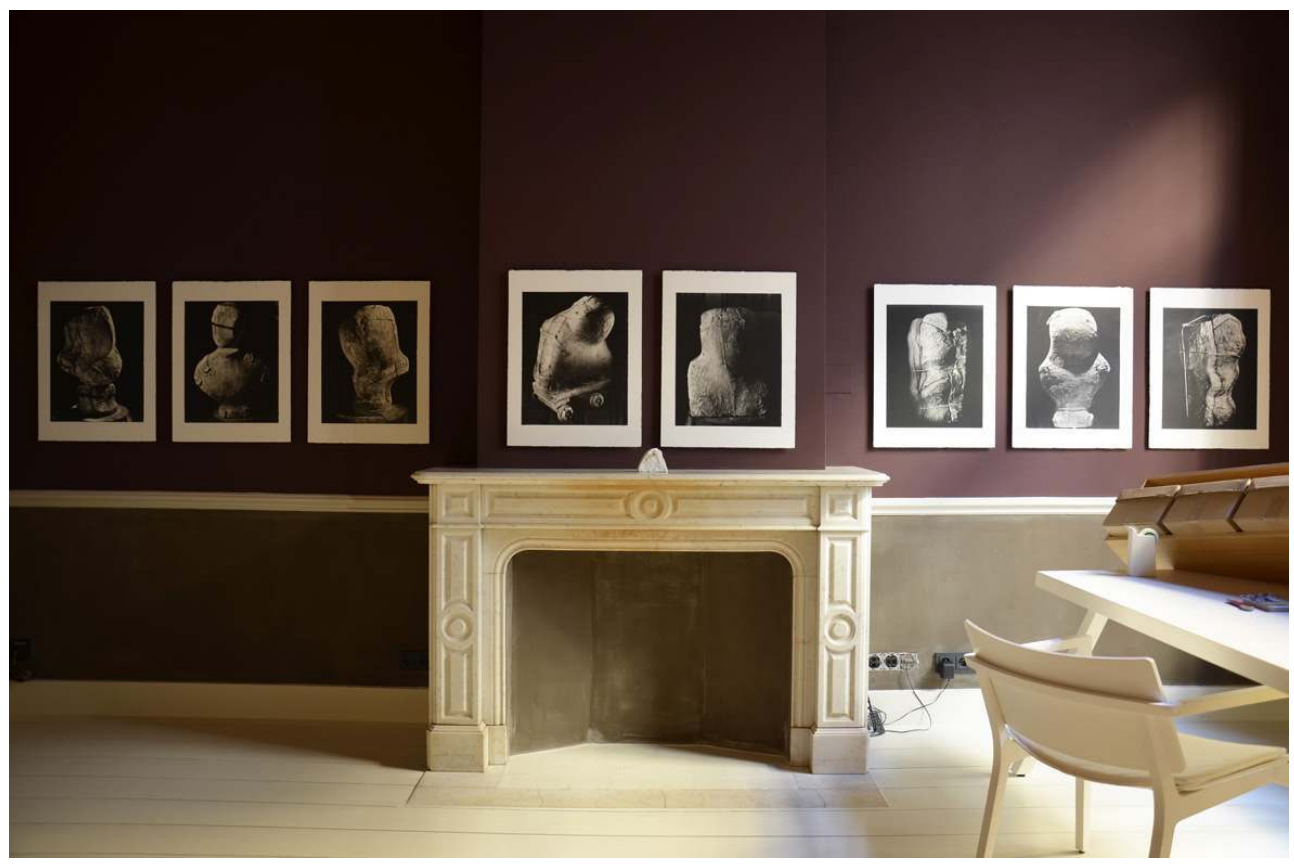

Albae umbrae «Les ombres blanches ». Photographie, 13 x émulsion sur papier BFK RIVES $210 \mathrm{~g}, 40 \mathrm{x}$ $50 \mathrm{~cm}$ (papier $50 \times 65 \mathrm{~cm}$ ).

(c) Martin Declève.

Figure 3

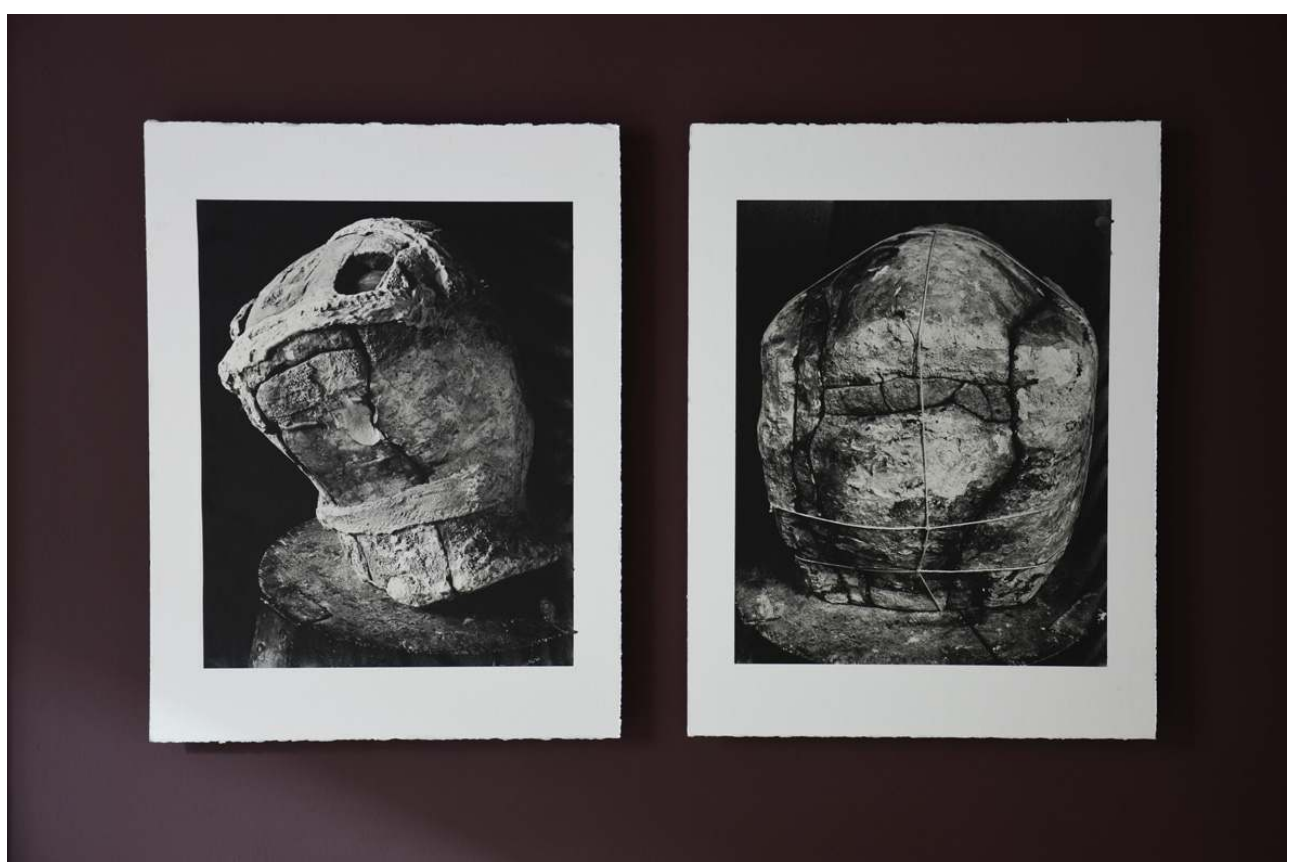

Brutus?, Inv. 628 et Antinoüs, Inv. 529. Photographies.

(C) Martin Declève. 


\section{Igneus est ollis vigor et caelestis origo}

9 Certains moules de bustes présentent la particularité d'avoir une chape dorsale en deux parties. Il est dès lors possible de ne laisser que celle qui couvre l'arrière de la tête, et de photographier le visage à travers l'orifice du cou, un peu comme on photographierait l'intérieur d'un vase. Les traits, s'inscrivant ainsi dans un orbe lumineux aux contours plus ou moins nets, ne sont pas sans évoquer les visages que les Anciens croyaient distinguer dans le disque de la lune. Imprimés sur verre, ils s'illuminent d'une lueur décalée qui provient du fonds devant lequel ils sont posés et, apparaissant davantage en relief qu'en creux, ils donnent la sensation d'émerger de l'on ne sait trop où, à la limite d'un dedans et d'un dehors qui ne cessent de se confondre dans une profondeur insaisissable.

10 Un des points de départ du travail avait été de placer l'objectif de l'appareil photographique à la place de l'âme, à la naissance du regard. Cela entendu bien sûr comme une métaphore visuelle, mais pas seulement lorsque l'on sait que le même mot désigne le morceau de bois sur lequel on modèle une statue et qui, une fois retiré après la fonte, laisse un vide. L'âme pour les Anciens, nommée de diverses manières (spiritus, animus, genius...), était conçue comme un souffle igné, situé essentiellement dans la tête, producteur de la semence, vecteur de la ressemblance et dont la face était le reflet visible. De même nature que ce souffle de feu était le regard, jeté par l'orifice des yeux pour aller palper le monde. Ceci explique que la photographie était à proprement parler inconcevable dans l'Antiquité - le rayon visuel sort de l'œil, la lumière n'étant qu'une condition du visible - et que le concept de ressemblance y était fort éloigné du nôtre, résultant plus d'une interaction entre le regard et la pensée que du relevé anthropométrique. Un passage des Florides de l'écrivain latin Apulée est éclairant à cet égard. Dressant un bref portrait d'Alexandre le Grand, Apulée rapporte comme trait principal de la personnalité du personnage le contrôle absolu qu'il avait de son image et le souci de la voir transmise avec la plus grande ressemblance partout dans l'empire. À cette fin Alexandre avait interdit que quiconque ne le représente à l'exception de trois artistes attitrés. C'est grâce à cela, ajoute Apulée, qu' « Alexandre a été le seul roi toujours ressemblant dans ses portraits : statues, tableaux, gravures ont reproduit partout très fidèlement l'énergie fougueuse du guerrier, le génie du héros magnifique, la beauté de la jeunesse en fleur, enfin la pureté d'un front découvert». On voit à quel point la ressemblance pour les Anciens est envisagée en tant qu'échange permanent et comme allant de soi entre le visible et l'invisible, toute physionomie étant le résultat d'un mélange inextricable entre les traits du visage et ceux de l'âme.

11 Les astres aussi participaient de cette même nature, mélange vital de souffle et de feu. Lâme leur fut associée et la métamorphose jadis réservée à quelques héros de la mythologie - le catastérisme - devint bientôt le lot commun de toute grande destinée, comme le laisse entendre Virgile dans l'Énéide dont est extraite la citation qui donne son titre à cette série. Il est drôle par ailleurs d'apprendre que le marbre le plus apprécié à Rome, le fameux marmor Lunense, venait des carrières de Luna (près de la Carrare actuelle), site dédié dès sa fondation à cet astre. 
Figure 4

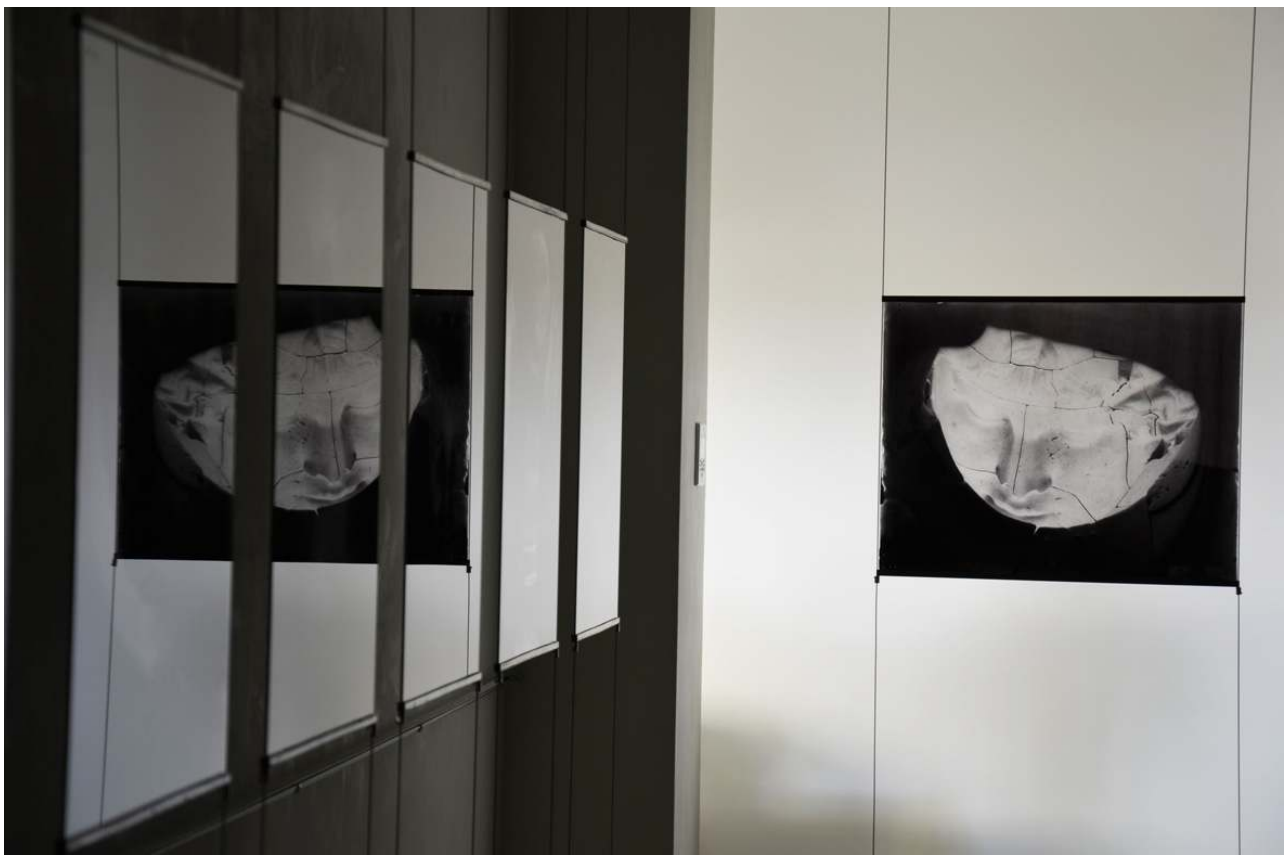

Igneus est ollis vigor et caelestis origo, « Leur force vitale vient du feu et leur origine est céleste ». Photographie, 7 x émulsion sur verre, 50 × 62 cm (détail : Inv. 279, Antinoüs).

(c) Martin Declève.

Figure 5

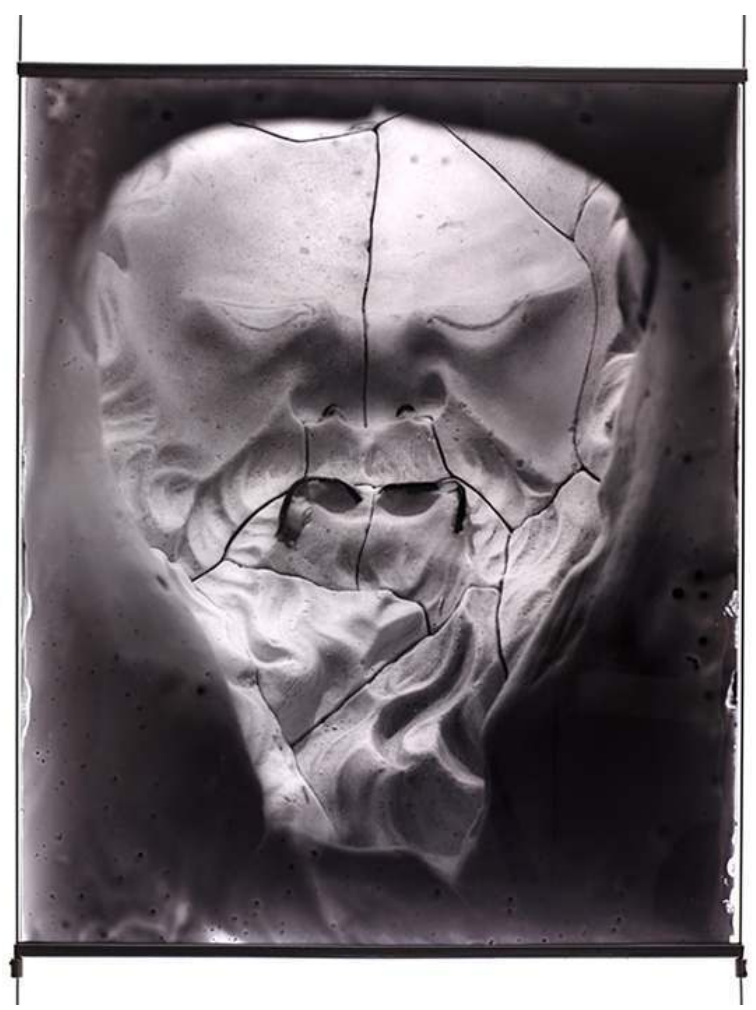

Socrate, Inv. 290. Photographie.

(c) Martin Declève. 
Figure 6

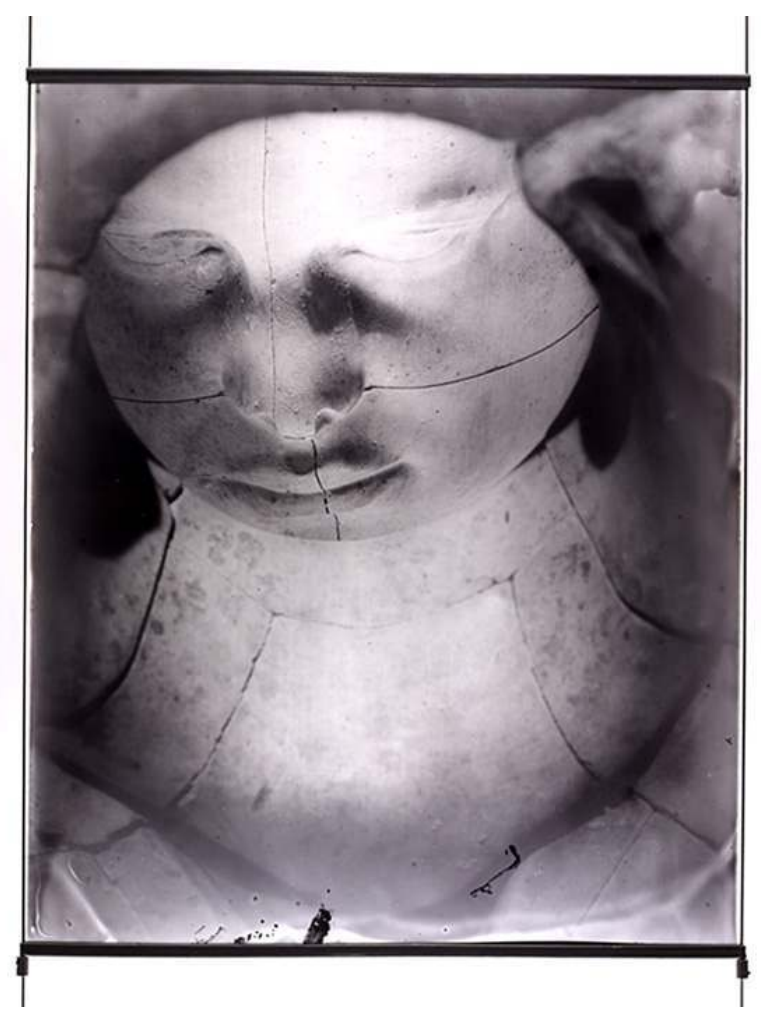

Dame romaine, Inv. 642. Photographie.

(c) Martin Declève.

\section{Atrium imaginarium}

Si je tente à partir d'ici de reprendre la genèse de ces images, les choses me paraissent aujourd'hui plus claires, même si cela n'est possible qu'au bénéfice d'une reconstruction comme c'est immanquablement le cas - a posteriori. Dès ses débuts - c'est déjà le cas chez W. H. Fox Talbot, l'inventeur du procédé positif/négatif, avec son buste de Patrocle dans son Pencil of Nature - la photographie a eu affaire à la sculpture et l'a prise comme motif de prédilection. La blancheur du sujet - sans toujours préciser s'il s'agit d'un original ou d'un moulage -, la possibilité de l'orienter ad libitum par rapport à la source lumineuse, le jeu possible avec les ombres, la variation de la distance, du cadrage, tout cela offrait et continue d'offrir au photographe un vaste champ d'expérimentation. Sans m'être alors intéressé à l'aspect historique de cette pratique, j'ai moi-même photographié, comme de nombreux photographes, des sculptures - particulièrement des sculptures antiques - à de multiples reprises, au gré de visites de musées, de visites de sites. Mais photographier une sculpture antique, c'est un peu comme photographier une ruine: le contexte contemporain de l'image (le musée la plupart du temps), les lacunes ou les restaurations de l'œuvre souvent indexent avant tout le passage du temps, l'absence. Problématique propre à la photographie s'il en est, ou du moins à bien des pratiques photographiques, dont je n'ai jamais pour ma part voulu faire le centre de mon travail. En revanche, créer des portraits photographiques à partir de moules m'est apparu comme un raccourci radical ouvrant sur d'autres horizons que celui que l'on reconnaît habituellement à ce 
médium. Retrouver une présence à partir d'une trace improbable - ces moules, rebut de l'Art, qui souvent, copies de copies, n'ont pas côtoyé l'original -, faire se lever un visage qui n'évoque pas immédiatement, par sa dégradation, par son style même, l'époque révolue qui fut la sienne mais qui, au contraire, par sa matérialité étrange, spectrale, sa fragmentation systématique et pourtant difficilement compréhensible pour les non connaisseurs, par le jeu, surtout, des creux et des reliefs dans lequel notre œil, trop prompt à recréer ce à quoi il est habitué, se perd, par ses traits inversés, enfin, comme dans un miroir, faire se lever un visage qui impose le reflet d'une présence. Ultime lueur des Anciens parmi nous, pareille au scintillement d'un astre depuis longtemps éteint.

Finalement, il s'agit d'appliquer une vieille règle mathématique, qui nous dit que moins et moins font plus. À la fin du $\mathrm{XIX}^{\mathrm{e}}$ siècle et au début $\mathrm{du} \mathrm{Xx}^{\mathrm{e}}$, alors que la querelle quant au statut artistique de la photographie faisait rage, certains sculpteurs, soupçonnés de mouler sur nature, se sont vu critiquer au motif qu'ils « remplaçaient un objet d'art par une photographie». En faisant couler dans ces moules bien moins que du plâtre, simplement de la lumière, j'ai voulu créer des images que seule la photographie pouvait nous donner à voir.

Figure 7

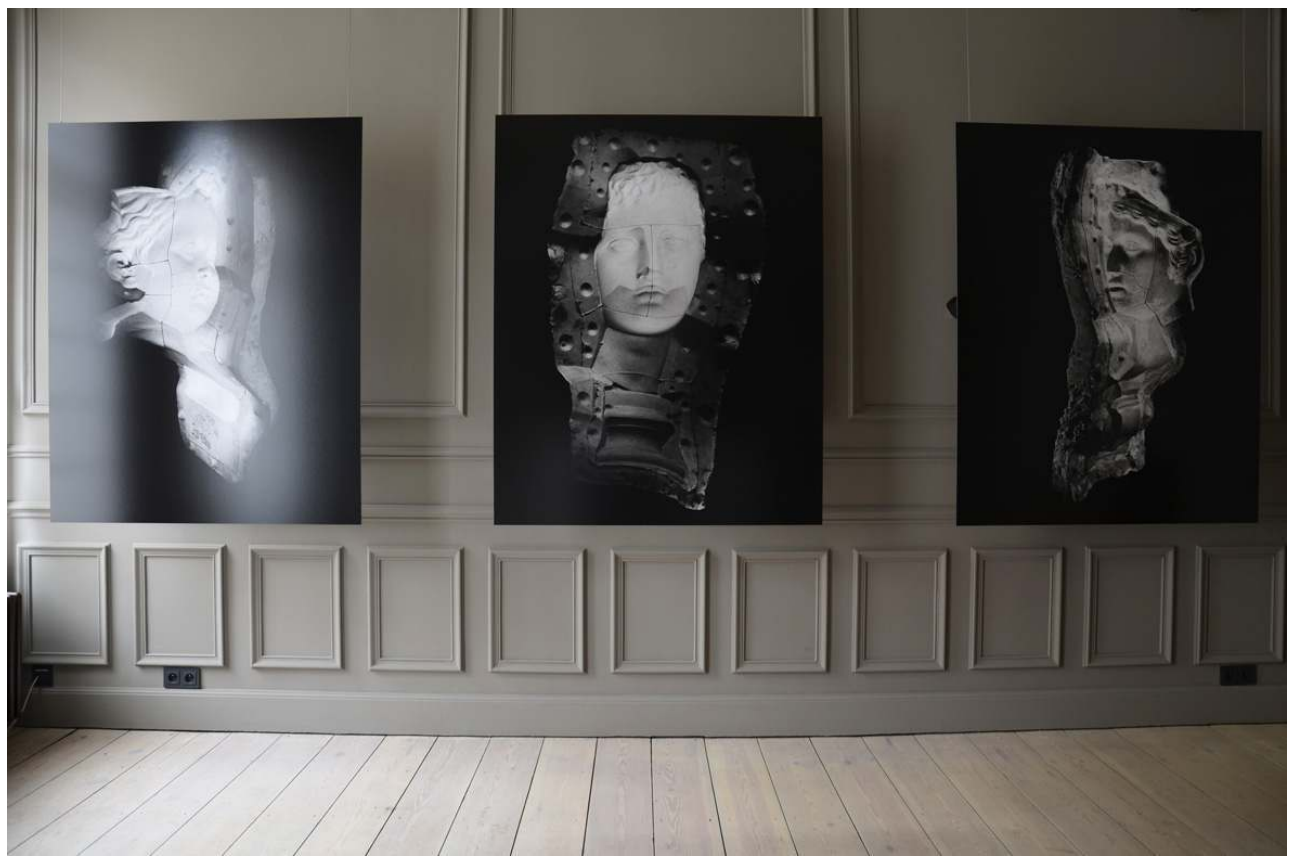

Atrium imaginarium, «L'atrium imaginaire ». Photographie, 6 x tirage argentique, $120 \times 150 \mathrm{~cm}$ (de gauche à droite : Inv. 028 [enfant], Inv. 111 [athlète], Inv. 357 [Sappho ?]).

(c) Martin Declève. 
Figure 8

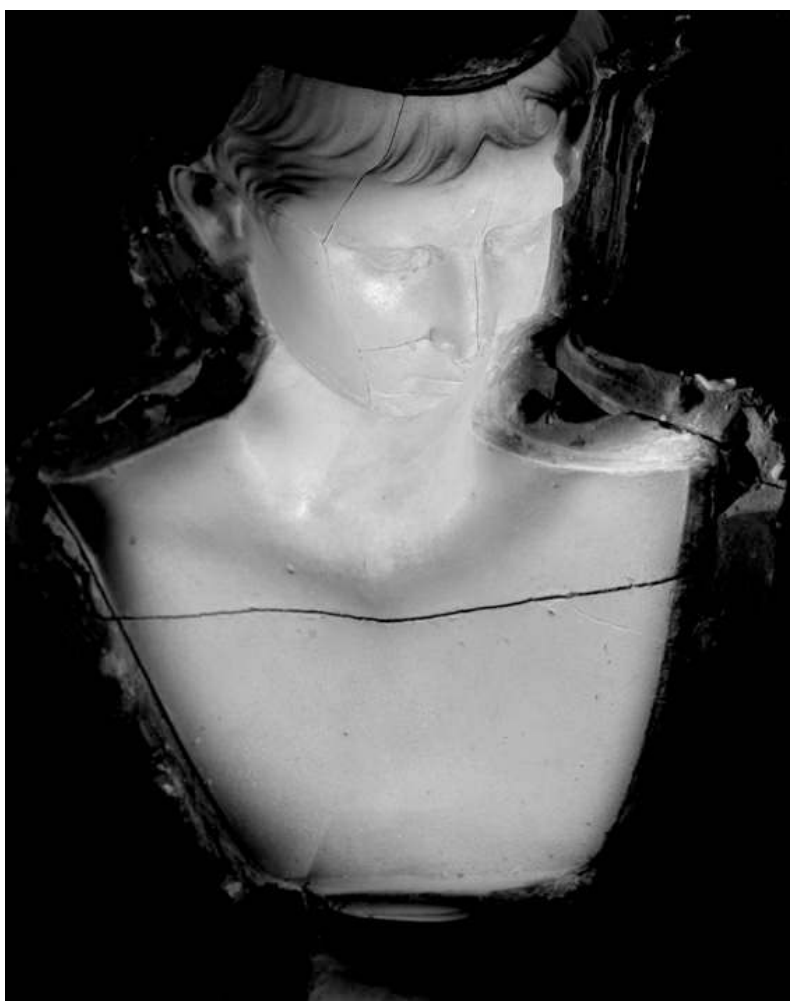

Auguste, Inv. 647. Photographie.

(c) Martin Declève. 


\section{Figure 9}

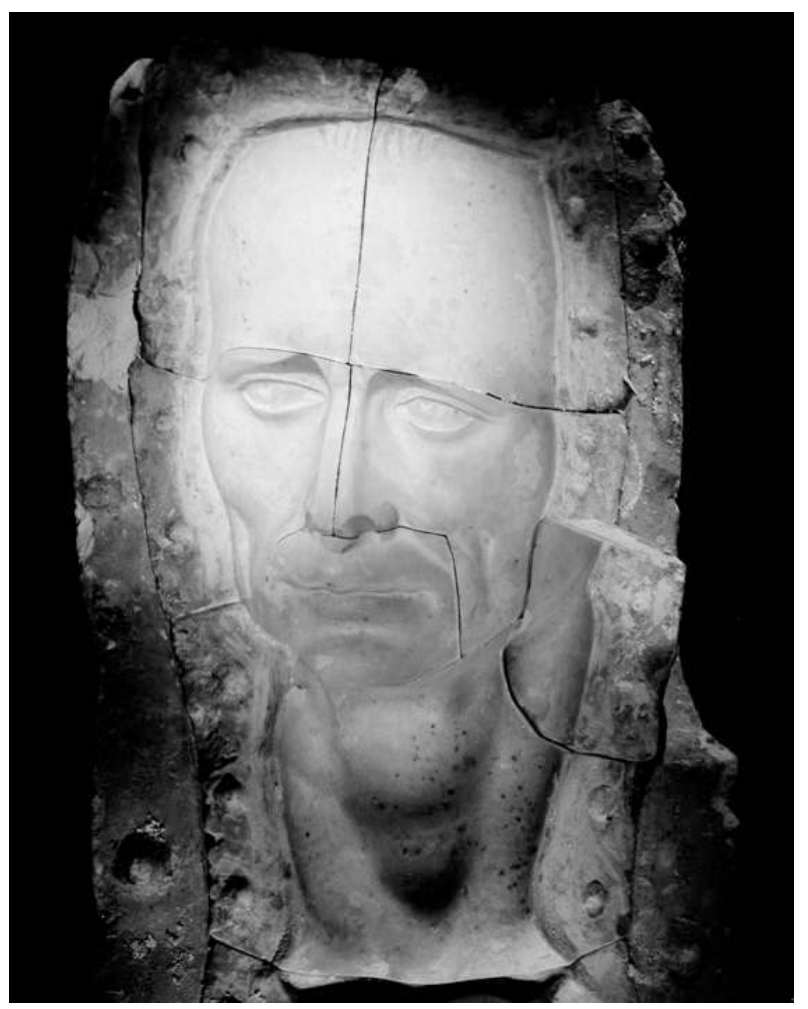

César, Inv. 652. Photographie.

(c) Martin Declève. 
Figure 10

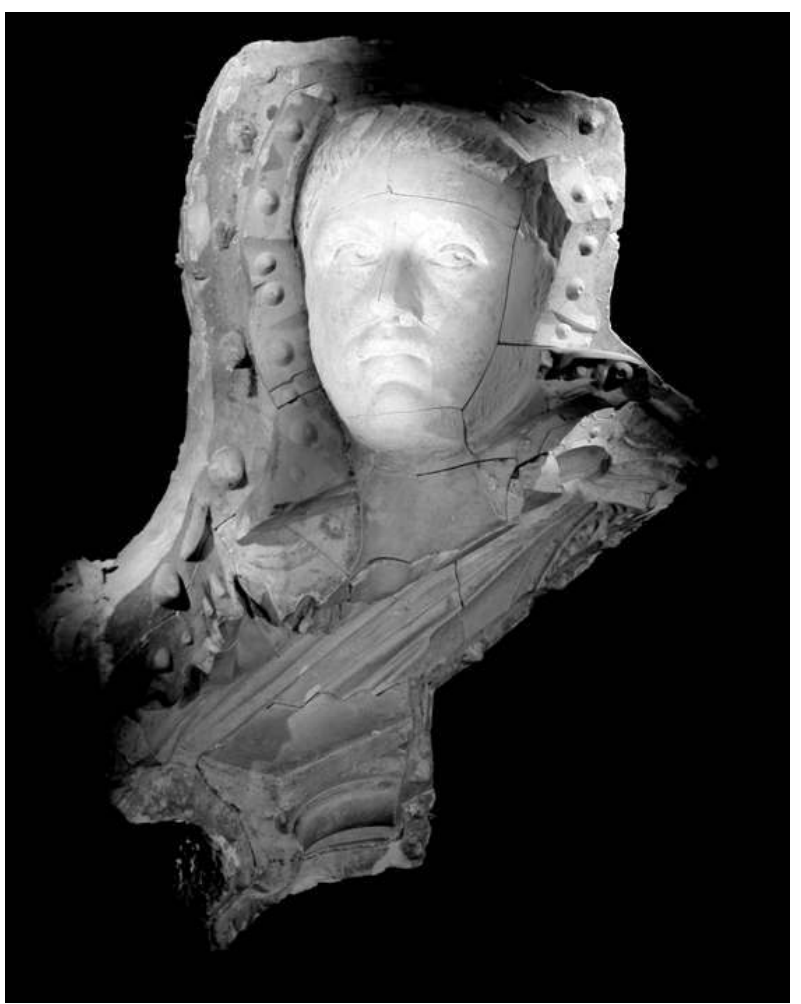

Gordien III, Inv. 624. Photographie.

(C) Martin Declève.

Né en Belgique en 1970, Martin Declève est photographe et cinéaste de formation. Son attrait pour l'Antiquité, né de sa pratique photographique sur les sites archéologiques et dans les musées, l'a amené à apprendre ces langues dites mortes - latin et grec ancien qu'il enseigne dans une école secondaire à Bruxelles. Depuis quelques années il a pu réduire son activité d'enseignant afin de se livrer davantage à la mise au point de projets qui rassemblent ses intérêts pour l'archéologie, la pensée antique, la tradition esthétique occidentale et la photographie.

\section{RÉSUMÉS}

À l'instar des peintres, les premiers photographes prirent pour modèles des moulages en plâtre d'œuvres antiques. Les galeries de moulages des grands musées constituaient le principal moyen pour se familiariser avec la sculpture classique. Avec le développement du tourisme culturel et les progrès de la photographie et de l'édition, les copies furent délaissées, sacrifiées au culte de l'original. Les galeries de moulages se vidèrent alors pour finalement souvent disparaître, entraînant avec elles bien des ateliers. Aujourd'hui que renaît un fort intérêt pour ce maillon de 
l'histoire de notre patrimoine que sont les moulages, c'est dans l'atelier des Musées royaux d'art et d'histoire que Martin Declève eut l'idée de poser son appareil pour photographier non plus des moulages, mais leurs moules, avec l'intuition qu'en coulant dans ceux-ci bien moins que du plâtre, simplement de la lumière, on obtiendrait des images que seule la photographie peut nous donner à voir. Par le jeu des creux et des reliefs, dans lequel notre œil, trop prompt à recréer ce à quoi il est habitué, se perd, il nous semble parfois qu'un illustre visage s'élève...

Like painters, the earliest photographers used, as models, the plaster casts of classical sculptures. The galleries of plaster moulds of the major museums were the main way for people to familiarize themselves with classical sculpture. With the development of cultural tourism, and the progress of photography and publishing, copies were abandoned, sacrificed at the altar of the cult of the original. The galleries of plaster-cast reproductions were no longer visited, and soon disappeared, along with the workshops that made the casts. Today, coinciding with a renewal of interest in this period in our heritage history, the photographer Martin Declève had the idea of taking photographs in the workshop of the royal museums of art and history. He was not interested in the finished casts but in the moulds, with the intuition that by pouring light into them, rather than plaster, it would be possible to produce pictures that photography alone can create. By playing on the effects of hollows and relief, our eye, used to making sense what it is used to, gets lost and sees famous faces emerge...

\section{INDEX}

Mots-clés : photographie, moule, moulage, portrait, image, buste, visage, antiquité, creux, lumière

Keywords : photography, mould, plaster cast, image bust, face, Antiquity, hollow, light

\section{AUTEUR \\ MARTIN DECLÈVE}

enseignant, photographe martin.decleve@gmail.com 